\title{
Récentes évolutions sur les mesures de débit par exploration du champ de vitesse au courantomètre
}

\begin{abstract}
Alexandre HAUET ${ }^{1}$, Lucas TORNATORE ${ }^{1}$
${ }^{1}$ EDF-DTG, 21 Avenue de l’Europe, BP 41, 38040 Grenoble, France, e-mail : alexandre.hauet@edf.fr

RÉSUMÉ. - En hydrométrie, la mesure de débit par exploration du champ de vitesse au courantomètre reste une méthode très largement utilisée. A EDF-DTG, cette méthode représente environ 30\% des 900 jaugeages réalisés chaque année. L'exploration du champ de vitesse est réalisée avec différents courantomètres, des historiques moulinets et micromoulinets mécaniques aux plus récents courantomètres acoustiques ou électromagnétiques. Les courantomètres acoustiques peuvent permettre de mesurer les trois composantes de vitesse, tandis que les courantomètres électromagnétiques sont peu sensibles à la végétation et peu invasifs dans l'écoulement.

Alors que les appareils de mesure évoluent, les méthodes de déploiement et le système qualité associé à leur utilisation a subi peu d'évolutions ces dernières décennies. Cet article présente deux évolutions récentes mises en place de façon opérationnelles à EDF-DTG. La première concerne le protocole de réalisation de la mesure, avec ajout de verticales intercalaires où l'on mesure uniquement la hauteur d'eau. Cette évolution permet de diminuer notablement l'incertitude du jaugeage sas pénaliser son temps de réalisation La seconde concerne l'étalonnage et le contrôle qualité des moulinets. On constate qu'un étalonnage périodique en laboratoire avec une équation par hélice n'est pas nécessaire, et qu'une équation unique par type d'hélice avec un contrôle périodique suffit.
\end{abstract}

Mots-clés : Courantomètres, Moulinets, Étalonnage

\section{Measuring discharge using the velocity area method: recent evolutions.}

\begin{abstract}
Discharge measurements using the velocity area method with a current meter is widely used in river hydrometry. At EDF-DTG, this method represents about $30 \%$ of the 900 gaugings performed each year. Different current meters can be used: mechanical propeller, acoustic or electromagnetic current meters. Those current meters are in constant development, but the measurement methods and the quality insurance related don 't have undergone significant changes those last decades. This paper presents two recent evolutions developed and operationally used at EDF-DTG. The first one deals with the measurement protocol, by adding verticals for which only the water depth is measured. The second one deals with the mechanical current meters calibration.
\end{abstract}

Key-words: Current meters, Mechanical current meters, Calibration.

\section{CONTEXTE}

En hydrométrie, la mesure de débit par exploration du champ de vitesse au courantomètre reste une méthode très largement utilisée. A EDF-DTG, cette méthode représente environ $30 \%$ des 900 jaugeages réalisés chaque année. L'exploration du champ de vitesse est réalisée avec différents courantomètres, des historiques moulinets et micro-moulinets mécaniques aux plus récents courantomètres acoustiques ou électromagnétiques. Les courantomètres acoustiques peuvent permettre de mesurer les trois composantes de vitesse, tandis que les courantomètres électromagnétiques sont peu sensibles à la végétation et moins intrusifs dans l'écoulement (instruments de petite taille).

Alors que les appareils de mesure évoluent, les méthodes de déploiement et le système qualité associé à leur utilisation a subi peu d'évolutions ces dernières décennies. Cet article présente deux évolutions récentes mises en place de façon opérationnelles à EDF-DTG. La première concerne le protocole de réalisation de la mesure, avec ajout de verticales intercalaires où l'on mesure uniquement la hauteur d'eau. La seconde concerne l'étalonnage et le contrôle qualité des moulinets.

\section{JAUGEAGE PAR EXPLORATION \\ DU CHAMP DE VITESSE \\ AU COURANTOMÈTRE : QUELQUES DÉFINITIONS}

Une définition précise d'un jaugeage par exploration du champ des vitesses peut être trouvée dans la Norme Iso 748 [Iso, 2009] ou dans la Charte Qualité de l'Hydrométrie [Forray et al., 1998]. Le principe d'un jaugeage par exploration du champ de vitesse au courantomètre est de répartir dans la section des verticales successives dans la profondeur desquelles des mesures de vitesses sont effectuées (Figure 1). La localisation des verticales, le nombre de mesures par verticale, leur localisation dans la profondeur et le temps de mesure doivent être déterminés avec soin.

Les mesures effectuées sur chaque verticale sont appliquées sur une largeur à droite et à gauche de cette verticale (Figure 1). Le nombre de points par verticale doit tenir compte à la fois de la profondeur, des variations verticales des vitesses et du matériel de mesure utilisé. 

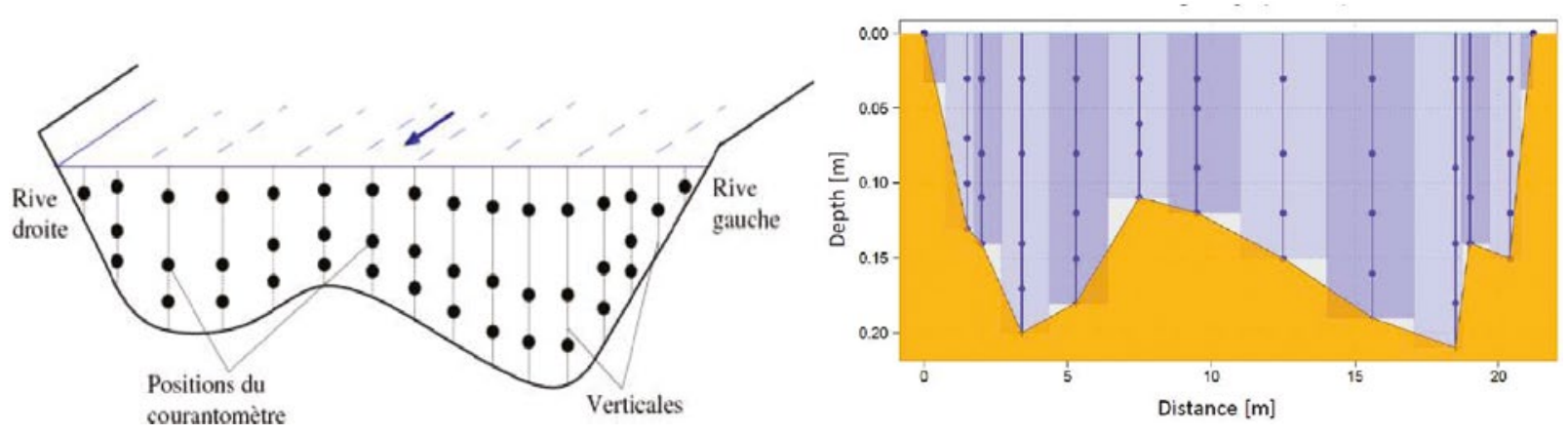

Figure 1 : Schéma de principe d'un jaugeage par exploration du champ des vitesses (à gauche, source : Charte Qualité de l'Hydrométrie) et illustration du dépouillement par section médiane (à droite, source [Despax et al., 2016]).

\section{EVOLUTION DU PROTOCOLE DE MESURE AU COURANTOMÈTRE : AJOUT DE LAMES D'EAU}

Les travaux concernant l'incertitude des jaugeages au courantomètres, qu'ils soient anciens comme la Norme Iso 748 ou récents comme les méthodes IVE [Kiang et al., 2009], Q+ [Le Coz et al., 2012] ou Flaure [Despax et al., 2016], montrent tous que la précision d'une mesure de débit par exploration du champ de vitesse repose essentiellement sur la finesse de la représentation de la bathymétrie et de la distribution des vitesses d'écoulement, c'est-à-dire le nombre de verticales de mesure. Le jaugeur doit trouver le compromis entre le nombre de verticales de mesure, la complexité de la section de jaugeage, et le temps de réalisation du jaugeage afin de rester dans des conditions d'écoulement permanent. Sur une section de jaugeage, les variations et gradients de vitesse dans l'écoulement sont moindres que les variations de la bathymétrie du fond. En effet, l'eau en tant que fluide adoucit les gradients et les vitesses évoluent graduellement. Les formes de fond, en revanche, peuvent montrer des gradients très importants. Une meilleure connaissance de la forme du fond permet donc un jaugeage de meilleure qualité.

La procédure de jaugeage mise en place à EDF-DTG consiste à intercaler, entre chaque verticale d'un jaugeage classique, une verticale dite "lame d'eau », où seul le tirant d'eau est mesuré. On arrive, dans l'exemple de la Figure 2 d'un jaugeage à la station EDF-DTG de Villard Loubière, à un jaugeage à 13 verticales complètes (mesure de la profondeur et mesure de vitesses) et 10 verticales « lame d'eau » (mesure de la profondeur). On constate que les verticales de type « lame d'eau » apportent une information complémentaire sur la bathymétrie intéressante.

Cette mesure rapide et simple permet de ne pas pénaliser le temps de jaugeage, tout en réduisant son incertitude. Une analyse a été menée sur 50 jaugeages de EDF-DTG réalisés avec le protocole «lame d'eau ». On a calculé l'incertitude de ces jaugeages grâce à la norme Iso 748 et à la méthode Flaure. Les jaugeages on été dépouillés avec et sans les verticales de type lame d'eau. Comme illustré dans le Tableau 1, on constate que l'ajout des verticales de type lame d'eau

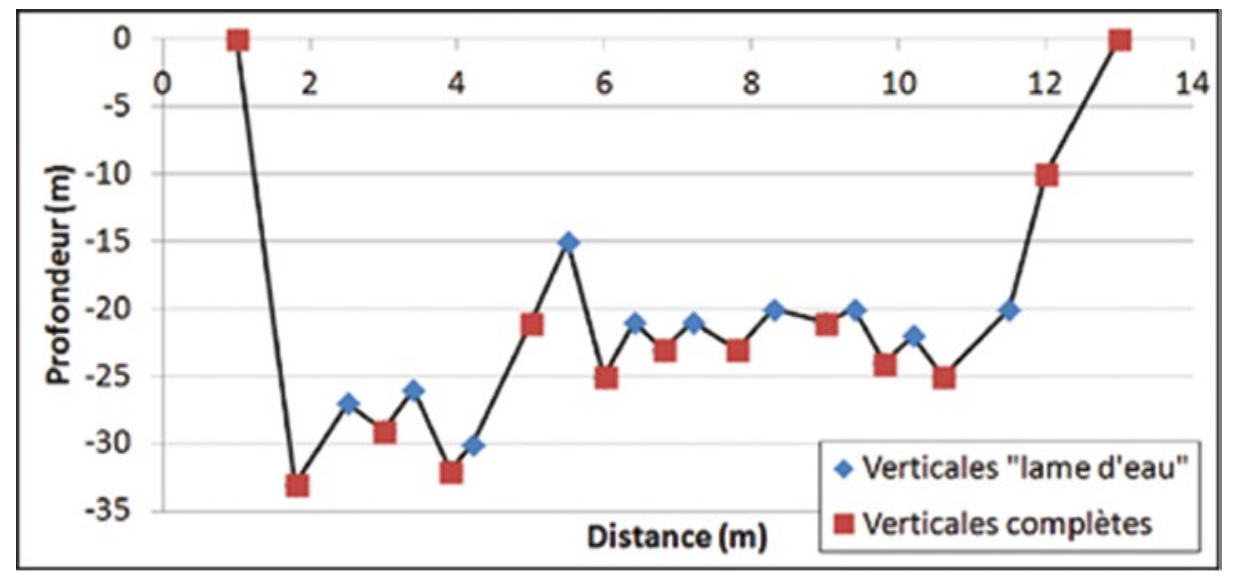

Figure 2 : Bathymétrie d'un jaugeage avec ajout de verticales de type «lame d'eau »

Tableau 1 : Incertitude (au seuil de confiance de $95 \%$ ) pour 50 jaugeages, avec et sans prise en compte des "lames d'eau »

\begin{tabular}{|l|l|l|l|}
\hline Incertitude & Moyenne & Min & Max \\
\hline Iso & $8 \%$ & $6 \%$ & $11 \%$ \\
\hline FLAURE avec lames d'eau & $7 \%$ & $3 \%$ & $17 \%$ \\
\hline FLAURE sans lames d'eau & $13 \%$ & $5 \%$ & $26 \%$ \\
\hline
\end{tabular}


permet de réduire fortement l'incertitude : en moyenne sur les 50 jaugeages, on passe de $13 \%$ à $7 \%$ d'incertitude (au seuil de confiance de $95 \%$ ).

\section{IV. ÉVOLUTION DU CONTRÔLE QUALITÉ DES MOULINETS HYDROMÉTRIQUES : ÉTALONNAGES ET TESTS D'ÉQUIPAGES MOBILES}

La seconde évolution concerne le contrôle qualité des moulinets hydrométriques. Un récent sondage au sein du Groupe Doppler Hydrométrie a montré que les pratiques de contrôle, dans la communauté des hydromètres, sont très variables : étalonnage à fréquence régulière chez le constructeur en bassin de carène, comparaison des moulinets opérationnels à un moulinet de référence, comparaison inter-laboratoire entre les moulinets, ou entre les moulinets et d'autres instruments (ADCP, autres courantomètres)...

Depuis 1998, EDF-DTG procède à un étalonnage systématique de toutes ses hélices en canal d'étalonnage, à une période de 3 ans. Un retour d'expérience montre que, pour tous les types d'hélice, la variabilité des modifications des équations est très faible, et de l'ordre de l'incertitude de l'étalonnage, comme illustré en Figure 3 pour les hélices de type 1 pour moulinets $\mathrm{C} 2$.

Les étalonnages et la mise à jour des équations d'hélice étant des actions coûteuses en temps, en argent, voire source d'erreur de saisie, il a été décidé d'attribuer une équation moyenne par type d'hélice, et de vérifier, avec une période de 5 ans, la conformité des hélices par rapport à l'équation moyenne et à un écart maximal tolérable (EMT). L'équation moyenne et l'EMT ont été calculés par EDF-DTG sur la base du retour d'expérience des étalonnages depuis 1998.

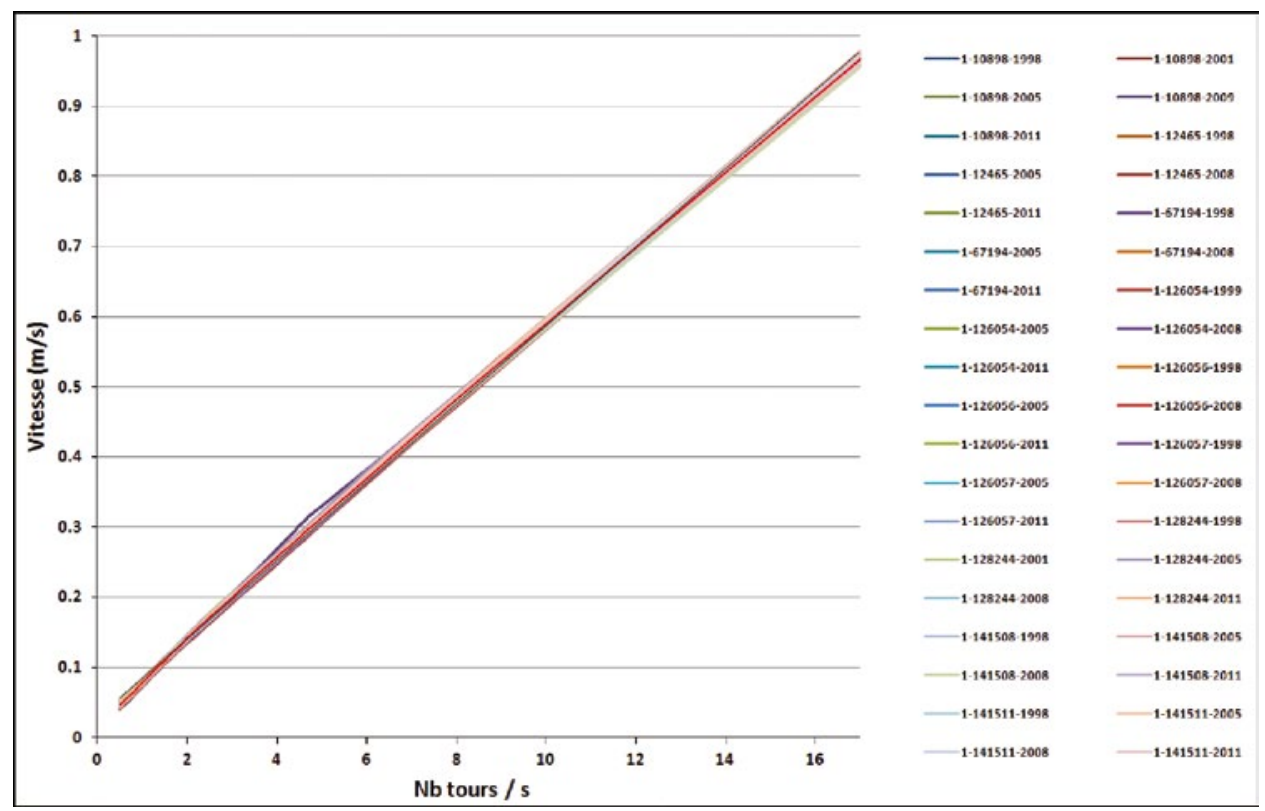

Figure 3 : Étalonnages de 10 hélices type 1 depuis 1998. Les résultats d'étalonnages sont très proches, et toutes les droites quasiment confondues. La légende indique le numéro de l'hélice et la date de l'étalonnage.

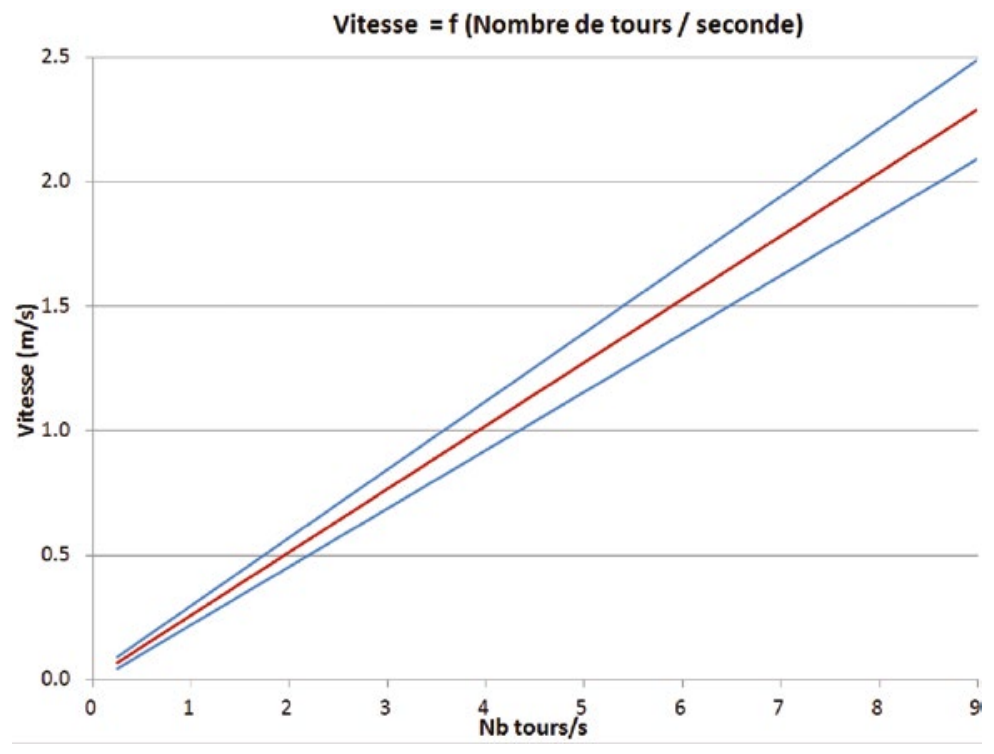

Figure 4 : Équation moyenne (en rouge) et EMT (en bleu) pour les moulinets C2 équipés d'hélices de type 3. 
L'équation moyenne par type d'hélice est la moyenne de toutes les équations d'étalonnage. L'EMT est calculé comme trois fois l'écart type de toutes les équations d'étalonnage, soit un intervalle à $98 \%$ (Figure 4 ).

\section{CONCLUSIONS}

Cet article présente deux évolutions récentes mises en place de façon opérationnelles à EDF-DTG concernant les jaugeages par exploration du champ de vitesse au courantomètre. La première concerne le protocole de réalisation de la mesure, avec ajout de verticales intercalaires ou l'on mesure uniquement la hauteur d'eau. Cette évolution permet de diminuer notablement l'incertitude du jaugeage sas pénaliser son temps de réalisation La seconde concerne l'étalonnage et le contrôle qualité des moulinets. On constate qu'un étalonnage périodique en laboratoire avec une équation par hélice n'est pas nécessaire, et qu'une équation unique par type d'hélice avec un contrôle périodique suffit.

\section{REFERENCES}

Despax A., Perret C., Garçon R., Hauet A., Belleville A., Le Coz J. ET FaVre A.-C. (2016) - Considering sampling strategy and cross-section complexity for estimating the uncertainty of discharge measurements using the velocity-area method. Journal of Hydrology, 533, 128-140.

Forray N., Auer J. C., Brochot J. F., Eraud Y., Favriau A., Ghio M.,Lallement C., Lang M., Odier M., and Scherer C. (1998) - Charte qualité de l'hydrométrie. Code de bonnes pratiques.

ISO (2009) - ISO 748:2009. Hydrometry: measurement of liquid flow in open channels using currentmeters or floats, $58 \mathrm{p}$.

Kiang J., Cohn T. et Mason R. (2009) - Quantifying uncertainty in discharge measurements: A new approach. In World Environmental and Water Resources Congress 2009. American Society of Civil Engineers, 8 p.

Le Coz J., Camenen B., Peyrard X. et Dramais G. (2012) - Uncertainty in open-channel discharges measured with the velocity-area method. Flow Measurement and Instrumentation, 26, 18-29. 